\title{
SUR LA TROMBIDIOSE HUMAINE EN URUGUAY
}

\author{
Par R.-V. TALICE
}

Nous avons publié dans ces Annales (1) une note sur la trombidiose des animaux en Uruguay. Nous disions que les «bichos colorados " (2), très abondants dans les champs incultes de l'Amérique du Sud, parasitent fréquemment l'homme.

Les lieux les plus favorables pour l'infestation sont les endroits humides (bois, forêts), où la végétation est constituée par des arbustes et de hautes herbes. C'est pendant l'été, de décembre à mars, que les "bichos colorados" se fixent de préférence sur l'homme : l'infestation a plus de chances de se produire dans les premières heures du matin ou dans les dernières du soir. Les larves attaquent d'abord les membres inférieurs; elles gagnent ensuite presque toutes les parties du corps (hanches, scrotum, prépuce, ventre, poitrine, dos, bras, cou), pour se fixer surtout au niveau de la face de flexion des plis articulaires et aux endroits où des obstacles s'opposent à leur libre passage. Ainsi, on trouve les larves fixées aux chevilles, où s'attache l'extrémité inférieure des culottes de cavaliers, au niveau des jarretelles, de la ceinture, au-dessous des seins chez la femme, autour du cou, etc.

Chez les personnes qui sont restées couchées dans les champs ou qui ont exposé leurs mains au contact des plantes, l'infestation commence par le torse ou les membres supérieurs et alors on voit exceptionnellement des piqûres sur les membres inférieurs.

Nous n'avons jamais observé de localisations sur le visage ou le cuir chevelu, comme il parait que c'est quelquefois le cas pour les « rougets » de la France, chez les enfants (3). Les localisations sur les parties découvertes et libres (mains par exemple) sont très rares. Les caractères cliniques de notre trombidiose humaine sont presque identiques à ceux qui ont été signalés par différents

(1) Sur quelques larves de Trombidin $x^{\prime}$ de l'Uruguay, parasites des animaux. Ann. de Parasit., VII, 1929, p. 483-493.

(2) Nous rappelerons que dans d'autres pays de l'Amérique latine on désigne sous un grand nombre de noms différents les petits acariens rouges parasites. Par exemple : en certaines régions du Brésil on les appelle "mouqui " ou "mouquen ", au Pérou " japa » ou " isangüe " ou " isango ", en Colombie " niabi ", au Mexique " thalsahuate " ou " thalzahualt ", à Cuba " colorado », dans les Antilles " bête rouge ", en Guyane « pou d'agouti », etc.

(3) P. Weiss a décrit aussi les localisations du " isangüe » (Trombicula) du Pérou, dans les oreilles chez les enfants (Informe presentado par la comisión organizada para estudiar la régión del Madre de Dios, Lima, 1924, p. 97).

Annales de Parasitologie, $\mathrm{T}$. VIII, $\mathrm{N}^{\mathrm{os}} 3-4 .-1^{\text {er }}$ juillet 1930 , p. $349-354$. 
auteurs pour la trombidiose de l'Europe et pour celle de l'Amérique du Nord.

Les personnes à peau délicate et fine, les femmes, les enfants, sont plus exposées aux atteintes des " bichos colorados ". Les paysans, même les enfants, présentent une immunité, ou plutôt ils sont accoutumés à ces acariens parce que sur eux on n'observe jamais de traces des piqûres. Au contraire, les habitants des villes, en général très sensibles, sont extrêmement importunés quand ils vont en villégiature. Mais, après avoir souffert des premières attaques, on est peu importuné le reste de la saison. L'usage des molletières et pantalons serrés au niveau des chevilles empêche, dans une certaine mesure, les attaques de ces désagréables parasites, qui provoquent des phénomènes très variables selon les sujets. Pendant les premières heures après l'infestation, l'acarien est fortement fixé sur place, rarement au niveau des poils, enfonçant son rostre armé sous la peau. Le corps reste à la surface, sous l'aspect d'un point rouge-orangé à peine perceptible. Nous n'avons pas constaté la pénétration dans les follicules pilaires, signalée par Ewing en Amérique du Nord. Plusieurs acariens sont souvent réunis sur un même point.

Ensuite, on voit rapidement apparaître sur les téguments toute une série de petites macules qui deviennent autant de papules, rosées le premier jour, rouges à la fin, circulaires, assez saillantes, isolées ou confluentes. Elles peuvent dépasser un centimètre de diamètre et sont entourées elles-mêmes d'une auréole rougeâtre. Rarement on peut voir des plaques irrégulières. En examinant attentivement ces papules, on retrouve facilement à leur centre le parasite qui les a produites. La piqûre elle-même n'est pas sentie, mais les phénomènes subjectifs commencent peu de temps après l'apparition des premiers symptômes objectifs. En effet, un ou deux jours après l'infestation, il se manifeste, dans tous les cas, un prurit intense, quelquefois intolérable, avec sensation pénible de cuisson. Les démangeaisons provoquées par les piqûres sont plus violentes quand on est couché et si elles sont nombreuses peuvent enlever tout sommeil. Il en résulte des grattages incessants, provoquant des lésions cutanées diverses : érythèmes, excoriations, vésicules, etc., et quelquefois des infections secondaires. Comme les " rougets ", nos "bichos colorados" ne vivent que quelques jours sur la peau de l'homme. Aussi, les troubles qu'ils occasionnent n'acquièrent-ils pas une gravité réelle. Les patients réclament rarement les soins d'un médecin. En règle générale, on constate le maximum de phénomènes subjectifs au troisième jour. L'affection ne dure pas plus d'une dizaine de jours. Mais il est intéressant de 
signaler le fait suivant: les phénomènes subjectifs persistent quelques jours après l'extraction de l'acarien de la papule.. On croit que ce fait résulte de l'action persistante des sécrétions irritantes que le parasite inocule sous les téguments de l'hôte (André).

D'autre part, ces parasites sont très difficiles à chasser malgré les bains savonneux, les lavages, les ablutions au vinaigre ou à l'alcool qu'on a conseillé. Nous n'avons pas observé l'apparition des érythèmes étendus comme dans la trombidiose européenne. On peut constater une légère réaction fébrile chez les enfants et des phénomènes nerveux d'excitation chez les prédisposés, mais les symptômes généraux ne s'observent que dans les cas d'infestation intense. Enfin, après guérison, il n'y a pas de cicatrices visibles sur la peau.

Identification des "Bichos colorados") de l'homme. - On sait que tous ces minuscules acariens (" rougets ", "bichos colorados ", " chiggers ", etc.), ne sont autre chose que des larves hexapodes d'acariens de la famille des Trombididæ. Cette famille se divise en plusieurs sous-familles dont quatre présentent un intérêt médical: Trombidinæ, Tarsoneminæ, Tetranychinæ et Cheyletinæ. La première seule contient des parasites habituels de l'homme; les trois autres ne renferment que des parasites accidentels (Brumpt). Nous rappelerons que les larves de Trombidinæ peuvent vivre en parasites, soit sur les arthropodes (insectes ou arachnides), soit sur des vertébrés. Les adultes, de couleur rouge vif comme les larves, et les nymphes sont libres et vivent sur les végétaux. On observe donc ici un cas de ce que M. Caullery a appelé parasitisme provisoire ou protélien.

Les Trombidinæ de l'Amérique du Sud sont encore peu connus. En Argentine et en Uruguay, il parait que le nom de "bicho colorado " a été appliqué en réalité à des types d'acariens bien différents les uns des autres. Comme nous le verrons, on les a considérés, soit comme des Trombidinæ, soit comme des Tetranychinæ, soit comme des Cheyletinæ. Nous croyons nécessaire de donner un bref aperçu historique sur cette question.

Weyenbergh, en 1876, a identifié le "bicho colorado " à une larve de Tetranychus molestissimus, vivant à la face inférieure des feuilles d'une plante appelée vulgairement " abrojo " (Xanthium macrocarpum). Mais, d'après Brèthes (cité par Lahille), le parasite en cause n'est pas une larve de Tetranychinæ et d'ailleurs le nom de l'espèce serait un nomen nudum parce qu'il n'a été accompagné, ni d'un dessin, ni d'une description (1). A ce propos, il nous sem-

(1) Nous n'avons pas pu lire la publication originale de Weyenbergh. 
ble intéressant d'ajouter une observation personnelle; au mois de janvier 1927, pendant notre été, nous avons eu l'occasion de traverser des champs incultes du département de San-José, où abondent les " abrojos ". Sous les feuilles de cette plante nous avons récolté une grande quantité de petits acariens rouges, du genre Tetranychus. Or, malgré les chances d'infestation auxquelles nous nous sommes volontairement exposé pendant la récolte, dans une saison très appropriée, nous n'avons constaté sur nos téguments, les jours suivants, aucune trace de piqûre. Cela ne serait pas le cas certainement s'il s'agissait de vrais " bichos colorados ", comme nous l'avons constaté maintes fois sur nous-même.

Holmberg, en 1876, disait déjà qu'il existe plusieurs espèces de " bichos colorados " capables de parasiter l'homme et décrit alors le Tetranychus irritans, le Trombidium sarcasticum, le T. arbicola et le $T$. deserticola (Lahille). Haller, en 1886, Belou, en 1903, et Greenway, en 1908, dans leurs travaux respectifs, souscrivent à l'opinion de Weyenbergh sans apporter d'arguments précis. Donc, Weyenbergh, Holmberg (en partie), Haller, Belou, Greenway ont considéré le " bicho colorado " comme une larve d'acarien de la famille Tetranychinæ. Or, les représentants du genre Tetranychus, en particulier, peuvent éventuellement piquer l'homme. Mais les piqûres de ces parasites produisent des accidents très légers et très passagers, parce qu'ils ne se fixent pas longtemps sur la peau. Lahille, par exemple, a décrit, comme parasite accidentel de l'homme, le Tetranychus silvestris qui vit sous les feuilles de luzerne et Artault a montré que Tetranychus telarius, agent de la maladie "rouge " de la vigne et du platane, peut passer sur l'homme. Mais sa piqûre laisse seulement une petite papule, blanche au centre, qui démange durant un quart d'heure (Brumpt). Il est par conséquent très improbable que l'agent de notre trombidiose soit un Tetranychiné.

Bruyant, en 1909, étudia un lot de "bichos colorados " fixés sur un épi de graminée (Distichlis scoparia Arrech.). Cet épi avait été envoyé par le savant botaniste Arrechavaleta de Montevideo. D'après Bruyant il s'agissait, dans ce cas, d'une espèce nouvelle d'acarien de la famille des Cheyletinæ, Pseudoleptus arrechavaletæ. Arrechavaleta croyait que les acariens fixés sur la graminée, étaient identiques à ceux qui s'attaquent généralement à l'homme chez nous, ce qui nous semble très improbable. Pour rendre irréfutable cette hypothèse, comme le dit aussi Bruyant, il aurait fallu recueillir les " bichos colorados " à l'état de parasitisme sur l'homme. Ce Cheyletiné est peut-être un parasite accidentel de l'homme, mais, pour les raisons déjà données pour les Tetrany- 
chinæ, nous croyons qu'il n'est pas non plus le vulgaire " bicho colorado ". En effet, les Cheyletinæ sont aussi considérés jusqu'ici comme des parasites accidentels, provoquant des accidents très légers et passagers.

Brèthes, en 1909, publie une description incomplète d'une larve d'un petit acarien rouge qu'il identifie au vrai parasite. Il ne lui a pas donné un nom parce qu'il pensait que l'adulte était déjà décrit et qu'il ne restait qu'à identifier les formes larvaires et adultes. " Ces identifications sont extrêmement difficiles et exigent l'élevage d'acariens dans le laboratoire, ce qui n'est pas toujours facile. Pour cette raison, les acarologues modernes, sans attendre le résultat lointain de longues observations, déterminent provisoirement les larves. Cette détermination permet au moins de les reconnaître parmi une quantité d'autres très semblables. " (Lahille).

Rauenbusch, en 1910, a été le premier à dire que le « bicho colorado " commun est un Trombidiné, mais il a confondu cette larve avec le " rouget " (Trombicula autumnalis) de l'Europe.

Lahille, en Argentine, en 1927, décrit le Microtrombidium brumpti, très semblable au Trombicula irritans, comme l'espèce vulgaire de " bicho colorado », agent de la trombidiose argentine. Plusieurs fois, pendant nos voyages en été à la campagne en Uruguay (spécialement au département de Paysandú), nous avons été attaqués par d'innombrables «bichos colorados». Chaque fois que nous avons fait l'extraction de parasites sur nous-mêmes, l'examen nous a toujours montré qu'il s'agissait de larves de Trombidinæ (mandibules en crochet aigu, palpes à 5 articles caractéristiques, yeux présents, etc.).

Cette année, dans le laboratoire du Prof. Brumpt, j'ai eu l'occasion d'examiner les échantillons des "bichos colorados" capturés par lui sur l'homme en Uruguay avec le Docteur Langeron, en février 1927. Toutes ces larves étaient des Trombidinæ appartenant à une seule espèce. Elles ont été étudiées par M. André et par moi et identifiées au Trombicula irritans.

Toutes les larves que nous avons examiné présentent, néanmoins, quelques différences avec le Trombicula irritans Riley, 1873. Pour cette raison, M. André a voulu compléter, dans la note qui suit la nôtre, la description de cette forme larvaire en la décrivant comme $T$. irritans Riley, var. uruguayensis.

Ces larves produisent chez nous tous les symptômes de la trombidiose, comme nous l'avons constaté sur l'homme après la récolte. Il est possible que d'autres acariens de la même famille soient capables de produire aussi les mêmes accidents. 


\section{RÉSUMÉ}

$1^{\circ}$ La trombidiose de l'homme, très répandue en été en Uruguay, offre des caractères étiologiques et cliniques très semblables à ceux de la trombidiose européenne et nord-américaine.

$2^{\circ}$ Le Tetranychus molestissimus décrit par Weyenbergh, et le Pseudoleptus arrechavaletæ décrit par Bruyant, si réellement ils attaquent l'homme, ne seraient, à notre avis, que des parasites accidentels. On n'a pas encore démontré, d'ailleurs, qu'un Tetranychiné ou un Cheyletiné soit capable d'occasionner les symptômes de la vraie trombidiose.

$3^{\circ}$ Les larves des petits acariens rouges, appelés vulgairement " bichos colorados ", agents de notre trombidiose, appartiennent à la sous-famille des Trombidinæ, comme les " rougets" de l'Europe et les " chiggers " de l'Amérique du nord.

$4^{\circ}$ Le Trombicula irritans Riley, 1873, var. uruguayensis André, 1930 (très semblable au Microtrombidium brumpti Lahille, 1927), trouvé par Brumpt et Langeron en Uruguay, reste la seule espèce bien décrite de ces Trombidinæ. On doit la considérer comme l'agent habituel de la trombidiose chez nous, de même que Trombicula autumnalis en France, par exemple. La forme adulte de la larve de ce parasite est encore inconnue.

\section{BIBLIOGRAPHIE}

Brumpt (E.). - Précis de Parasilologie, $4^{\circ}$ éd., Paris, Masson, 1927.

Bruyant (L.). - Pseudoleptus arrechavalete n. gen. n. sp., nouvel acarien cheylétinế de l'Uruguay. Zoologischer Anzeiger, XXXVIII, 1911, nos 14-15.

Binet (E.). - Les trombidions parasites de l'homme. Revue des Sc. méd., pharm. et vétér., I, 1898, p. 40-42.

Bambaren (C.-A.). - Estudio de la larva parásita de un ácaro (Leptus japa Ribeyro y Bambaren) de la montaña del Perú. Crónica médica, XXXIX, 1922, p. 281-287. Analysé dans Bull. Inst. Pasteur, XXIII, 1923, p.

Ewing (H.-E.). -- Studies of the biology and control of the chiggers. United States Dep. of. Agric., Bull. n 986, 1921, 19 p.

Garzón Maceda (F.). - La Parasitología humana en la República Argentina. Córdoba, 1915, 62 p.

Lahille (L.). - Nota sobre alganos ácaros del país. 3e Reun. Soc. argentina patol. region. del norte, Tucuman, 1927. Bol. Inst. Clin. quir., Buenos-Aires, 1927, p. $662-680$.

Oudemans (A. C.). - Die bis jetzt bekannten Larven von Thrombidida und Erythræida. Zoolog. Jahrb., 1912, suppl. XIV, 230 p.

Toldt (K.). - Ueber die Trombidiase in Alpen. Wien. klin. Woch., XXXVI, $1923, \mathrm{n}^{\circ} 33$.

Vitzthum (G.). - Zoologische Acarusstudien. Zeitschr.f. Parasitenkunde, I, 1928, p. $1-23$.

- Systematische Betrachtungen zur der Trombidiose. Zeitschr. f. Parasitenkunde, II, 1929, p. 223-247.

Weiss (P.), Rojas (H.) et Guzmán Barron (A.). - Informe de la comisión organizada para estudiar la región del Madre de Dios, Lima, 1924, 113 p.

Warburton (G.). - The harvest bug. Parasitology, XX, 1928, p. 228-236. 